\title{
The novel polysaccharide deacetylase homologue Pdi contributes to virulence of the aquatic pathogen Streptococcus iniae
}

Correspondence

John T. Buchanan

jtbuchanan@gmail.com

Received 14 February 2009

Revised 7 September 2009

Accepted 14 September 2009
Carlo J. E. Milani, ${ }^{1} \dagger$ Ramy K. Aziz, ${ }^{2} \dagger$ Jeffrey B. Locke, ${ }^{1}$ Samira Dahesh, ${ }^{1}$ Victor Nizet ${ }^{1,3}$ and John T. Buchanan ${ }^{1,4}$

\author{
${ }^{1}$ Department of Pediatrics, University of California, San Diego, 9500 Gilman Drive, MC 0687 , \\ La Jolla, CA 92093-0687, USA
}

${ }^{2}$ Department of Microbiology and Immunology, Faculty of Pharmacy, Cairo University, 11562 Cairo, Egypt

${ }^{3}$ Skaggs School of Pharmacy and Pharmaceutical Sciences, University of California, San Diego, 9500 Gilman Drive, MC 0657, La Jolla, CA 92093-0657, USA

${ }^{4}$ Aqua Bounty Technologies, 8395 Camino Santa Fe, Suite E, San Diego, CA 92121, USA

The aquatic zoonotic pathogen Streptococcus iniae represents a threat to the worldwide aquaculture industry and poses a risk to humans who handle raw fish. Because little is known about the mechanisms of $S$. iniae pathogenesis or virulence factors, we established a highthroughput system combining whole-genome pyrosequencing and transposon mutagenesis that allowed us to identify virulence proteins, including Pdi, the polysaccharide deacetylase of $S$. iniae, that we describe here. Using bioinformatics tools, we identified a highly conserved signature motif in Pdi that is also conserved in the peptidoglycan deacetylase PgdA protein family. A $\Delta$ pdi mutant was attenuated for virulence in the hybrid striped bass model and for survival in whole fish blood. Moreover, Pdi was found to promote bacterial resistance to lysozyme killing and the ability to adhere to and invade epithelial cells. On the other hand, there was no difference in the autolytic potential, resistance to oxidative killing or resistance to cationic antimicrobial peptides between $S$. iniae wild-type and $\Delta p d i$. In conclusion, we have demonstrated that pdi is involved in $S$. iniae adherence and invasion, lysozyme resistance and survival in fish blood, and have shown that pdi plays a role in the pathogenesis of $S$. iniae. Identification of Pdi and other $S$. iniae virulence proteins is a necessary initial step towards the development of appropriate preventive and therapeutic measures against diseases and economic losses caused by this pathogen.

\section{INTRODUCTION}

In the past few decades, the pathogen Streptococcus iniae has emerged as a major hindrance to aquaculture operations worldwide (Agnew \& Barnes, 2007), causing economic losses measured in hundreds of millions of dollars annually. In addition, S. iniae has established itself

†These authors contributed equally to this work.

Abbreviations: AMP, antimicrobial peptide; HSB, hybrid striped bass, i.p., intraperitoneal; PIA, polysaccharide intercellular adhesin, WT, wild-type.

The GenBank/EMBL/DDBJ accession number for the pdi sequence of Streptococcus iniae is FJ664396.

Four supplementary figures, showing a schematic representation of allelic replacement of the $S$. iniae pdi gene with cat and the results of comparison of haemolytic activity, culture buoyancy and growth analysis, investigation into the autolytic potential, and resistance to oxidative killing and antimicrobial peptides of WT S. iniae and the $\Delta$ pdi mutant, are available with the online version of this paper. as a zoonotic risk, especially in areas of the world that preferentially prepare and consume raw fish (Lau et al., 2003). To date, at least 25 human cases of invasive streptococcal infection attributed to $S$. iniae have been confirmed in the USA, Canada, China and Taiwan, many of which were in immunocompromised patients (Agnew \& Barnes, 2007; Sun et al., 2007; Weinstein et al., 1997); since there is currently no prospective epidemiological surveillance for human $S$. iniae infections, the true number may be much higher (Facklam et al., 2005; Lau et al., 2006).

In spite of the economic and human health risks that $S$. iniae presents, a fully assembled genome sequence for this emerging pathogen is not yet available, and very little is known about its disease mechanisms. To better understand the molecular genetic basis of virulence in this versatile pathogen, we created a library of randomly generated transposon mutants that we screened for virulence 
attenuation in hybrid striped bass (HSB; Morone chrysops $\times$ Morone saxatilis) (Buchanan et al., 2005). The analysis and study of mutants from this library, along with candidate gene approaches, has previously served to elucidate virulence factors contributing to $S$. iniae pathogenicity in fish, including phosphoglucomutase (Buchanan et al., 2005), streptolysin S (Fuller et al., 2002; Locke et al., 2007a) and capsular polysaccharide (Barnes et al., 2003; Locke et al., 2007a; Lowe et al., 2007; Miller \& Neely, 2005). In addition, recent whole-genome pyrosequencing of $S$. iniae strain K288 has uncovered an mga-like pathogenicity locus (Locke et al., 2008), which includes an M-like protein virulence factor similar to the fibrinogen-binding proteins discovered in S. iniae strains QMA0076 and QMA0131 (Baiano et al., 2008).

There has been a recent appreciation of the role of bacterial deacetylases in virulence. In Staphylococcus epidermidis, exopolysaccharide deacetylation has been shown to be crucial for biofilm formation, surface colonization, resistance to neutrophil phagocytosis, and resistance to cationic antimicrobial peptides (AMPs) (Vuong et al., 2004). Protection against host defences by peptidoglycan deacetylases has also been reported for Listeria monocytogenes (Boneca et al., 2007) and Streptococcus pneumoniae (Vollmer \& Tomasz, 2000, 2002). In both cases, modification of the cell wall by a peptidoglycan deacetylase (PgdA) provides increased resistance to lysozyme and is essential for full virulence of the pathogen in a small animal model of infection.

In this study, transposon mutagenesis coupled with wholegenome pyrosequencing allowed us to identify a polysaccharide deacetylase gene, $p d i$ (polysaccharide deacetylase of $S$. iniae), implicated in S. iniae virulence. We provide an analysis of the conserved amino acid residues of Pdi homologues in representative Gram-positive bacteria. By targeted mutagenesis, we confirm a contribution of the $p d i$ gene to $S$. iniae virulence in a fish infection model, and use in vitro assays to probe potential mechanistic associations of pdi with augmented lysozyme resistance, enhanced blood survival and increased adherence to and invasion of fish epithelial cells.

\section{METHODS}

Bacterial strains, culture, transformations, and DNA techniques. Wild-type (WT) S. iniae strain K288 was isolated from the brain of a diseased HSB at the Kent SeaTech facility in Mecca, CA, USA (Buchanan et al., 2005). Escherichia coli used in cloning was grown on Luria agar at $37{ }^{\circ} \mathrm{C}$ with antibiotic selection of $500 \mu \mathrm{g}$ erythromycin $\mathrm{ml}^{-1}(\mathrm{Erm}), 100 \mu \mathrm{g}$ spectinomycin $\mathrm{ml}^{-1}$, and $20 \mu \mathrm{g}$ chloramphenicol $(\mathrm{Cm}) \mathrm{ml}^{-1}$, when appropriate. Mach 1 chemically competent E. coli (Invitrogen) and MC1061 electrocompetent E. coli, used for plasmid propagation during transformation, were recovered through growth at $30^{\circ} \mathrm{C}$, or $37^{\circ} \mathrm{C}$, when appropriate, with shaking in Super Optimal Broth with Catabolite Repression (S.O.C.) medium (Invitrogen). A PureLink Quick plasmid miniprep kit (Invitrogen) was used for plasmid isolation. S. iniae was grown in Todd-Hewitt broth (THB) without shaking or on Todd-Hewitt agar (THA) plates at $30{ }^{\circ} \mathrm{C}$, unless otherwise indicated, with antibiotic selection of $2 \mu \mathrm{g}$ $\mathrm{Cm} \mathrm{ml}{ }^{-1}$, or $5 \mu \mathrm{g} \mathrm{Erm} \mathrm{ml}^{-1}$ when required. The DNA Easy Tissue kit (Qiagen) was used for the isolation of S. iniae genomic DNA. Dilution plating on THA was used for enumeration of c.f.u. for in vitro assays. $\beta$-haemolytic activity was assessed on sheep blood agar plates (tryptic soy agar with $5 \%$ sheep red blood cells added) as previously described (Locke et al., 2007a). In all assays, overnight cultures of $S$. iniae were diluted $1: 10$ in fresh THB and grown to midexponential phase $\left(\mathrm{OD}_{600} 0.40\right)$, unless otherwise stated. The bacteria were rendered electrocompetent for transformation by the addition of $0.6 \%$ glycine to THB as previously described (Locke et al., 2007a).

Cell line and culture conditions. The WBE27 white bass embryonic epithelial cell line (ATCC CRL-2773) (Shimizu et al., 2003) was grown at $28{ }^{\circ} \mathrm{C}$ with $5 \% \mathrm{CO}_{2}$. Cells were passaged less than 10 times before use in experiments and were maintained in $125 \mathrm{ml}$ tissue culture flasks in Dulbecco's Modified Eagle's Medium (DMEM; Gibco) containing $10 \%$ heat-inactivated fetal bovine serum, (FBS; Gibco).

Transposon mutagenesis. The temperature-sensitive plasmid pTV1OK containing transposon Tn917 was used for transposon mutagenesis of strain K288 as previously described (Buchanan et al., 2005). Briefly, individual colonies of K288 transformed with pTV1OK were inoculated in THB plus kanamycin $\left(500 \mu \mathrm{g} \mathrm{ml}^{-1}\right)$ and grown to $\mathrm{OD}_{600} 0.90$ at a permissive temperature $\left(30^{\circ} \mathrm{C}\right)$ for plasmid replication. Cultures were diluted $1: 100$ in THB with Erm $(5 \mu \mathrm{g}$ $\left.\mathrm{ml}^{-1}\right)$ and grown at a non-permissive temperature $\left(37^{\circ} \mathrm{C}\right)$ to $\mathrm{OD}_{600}$ 0.90 , then plated on THA with Erm for the isolation of candidate insertion mutants. Single random transposon insertions were verified through Southern blot analysis of a subset of the library mutants. Over 1000 mutants were screened for attenuation in vivo in an HSB challenge model by intraperitoneal (i.p.) injection. (Buchanan et al., 2005). Screening the transposon library in this manner revealed over 40 attenuated mutants, including mutant TnM7.

Identification of mutated genes. The Tn917 insertion sites were identified in the candidate insertion mutants through single-primer PCR and direct genomic sequencing. PCR fragments extending out from the end of the transposon were created at random via singleprimer PCR by the use of the internal forward primer $5^{\prime}$ AATCTGTACCACTAATAACTC-3'. The external forward primer 5'-AATGTACAAAATAACAGCGA-3' was used for sequencing the PCR fragments.

Sequence assembly and localization of pdi. Contiguous sequences used for ORF determination and bioinformatics analysis were created from an automated assembly of pyrosequencing results (454 Life Sciences, Roche). This primary assembly was generated by the Phred/Phrap/Consed suite (Gordon et al., 1998) and resulted in 1865 contigs ranging in size from $51 \mathrm{bp}$ to $22 \mathrm{~kb}$ (Locke et al., 2008). Without further assembly, the 1865 contigs were used for building a local S. iniae K288 BLAST database, and then a local copy of BLAST version 2.2.14 (Altschul et al., 1997) was used for finding the contiguous sequences matching the pdi-deficient transposon mutant, TnM7, in S. iniae K288. Vector NTI (Invitrogen) was used in ORF identification and graphic representation of chromosomal positioning of pdi (GenBank accession number: FJ664396).

Bioinformatics and phylogenetic analysis of Pdi and its homologous proteins. Pdi homologues in different microbial genomes were retrieved from the National Microbial Pathogen Data Resource, (NMPDR; McNeil et al., 2007), by the use of the SEED similarity tool (Overbeek et al., 2005). SignalP algorithm (version 3.0) was used to screen the protein sequences for leader peptides (Bendtsen et al., 2004). Several tools were used for motif finding and protein family analysis, including InterPro (Apweiler et al., 2001), 
Pfam (Finn et al., 2008), and FigFam (Aziz et al., 2008). Further sequence analysis was performed on the Biology Workbench analysis platform (Subramaniam, 1998), URL: http://workbench.sdsc.edu). The specific Workbench tools that were used are: CLUSTAL W (Higgins et al., 1996; Thompson et al., 1994) for sequence alignment, PHYLIP (Felsenstein, 1997) for primary phylogenetic analysis, and BoxShade for sequence colouring. For phylogenetic analysis by the maximumlikelihood method, we used the Phylogeny.fr online server (Dereeper et al., 2008) and we edited the tree using the FigTree software (http:// tree.bio.ed.ac.uk/software/figtree). The Baylor S. iniae genome project (http://www.hgsc.bcm.tmc.edu/bcm/blast/microbialblast.cgi?organism $=$ Siniae) was utilized for comparative analyses.

Allelic exchange mutagenesis of the pdi locus. To confirm that the attenuation of TnM7 was a result of the inactivation of the pdi gene rather than a polar effect of transposon insertion or spontaneous mutation elsewhere in the chromosome, we generated an isogenic $\Delta p d i$ mutant by precise in-frame allelic replacement of $p d i$ with chloramphenicol acetyltransferase (cat) in the WT S. iniae strain K288 (see Supplementary Fig. S1 available with the online version of this paper). Allelic exchange mutagenesis was carried out as previously described (Locke et al., 2007a). Briefly, PCR was used to amplify $\sim 400$ bp upstream and $\sim 200$ bp downstream of the targeted S. iniae chromosomal gene region. Primers adjacent to the upstream and downstream regions of pdi were constructed with 25 bp $5^{\prime}$ extensions corresponding to the $5^{\prime}$ and $3^{\prime}$ ends of the cat gene from pACYC (Nakano et al., 1995). Fusion PCR was then used to combine upstream and downstream products with a $660 \mathrm{bp}$ amplicon of the cat gene (Buchanan et al., 2006). The fusion product resulting from this PCR contained an in-frame substitution of $p d i$ with cat and was subcloned into the Gateway entry vector pCR8/GW/TOPO. This vector was then used to transform chemically competent Mach $1 \mathrm{E}$. coli cells (Invitrogen). Plasmid DNA was extracted, and the fusion PCR amplicon was transferred into the temperature-sensitive knockout vector pKODestErm (Locke et al., 2007a) via an attL-attR (LR) recombination reaction to create the knockout plasmid pKOpdi. After its propagation in MC1061 E. coli, the pKOpdi construct was introduced into WT S. iniae through electroporation. Transformants were identified at $30{ }^{\circ} \mathrm{C}$ by Erm selection and shifted to $37{ }^{\circ} \mathrm{C}$ (a nonpermissive temperature for plasmid replication). Differential antibiotic selection of $\mathrm{Cm}^{\mathrm{r}}$ and $\mathrm{Erm}^{\mathrm{s}}$ allowed identification of candidate colonies as potential allelic exchange mutants. Targeted in-frame replacement was confirmed through PCR-documenting the desired insertion of cat and the absence of the pdi sequence in chromosomal DNA isolated from the $\Delta p d i$ mutant.

In vivo fish challenges. Groups of 10 HSB fingerlings $(\sim 15.8 \pm 3.7 \mathrm{~g})$ were challenged through i.p. injection with either WT or mutant $S$. iniae bacteria from mid-exponential-phase cultures. Injection with a 26.5 gauge needle was used to deliver either $1 \times 10^{5}$ or $1 \times 10^{4}$ c.f.u. in $50 \mu$ volumes of PBS. Fish were held with aeration and flow-through water at $24-27{ }^{\circ} \mathrm{C}$ for 7 days post-injection, and their survival was monitored daily.

Whole blood survival. Heparinized blood collected for bacterial survival assays was taken and pooled from four to six HSB fingerlings. Fresh blood was collected via caudal vein blood draw. A $75 \mu \mathrm{l}$ sample of blood was added to $2 \mathrm{ml}$ siliconized plastic centrifuge tubes containing approximately $5 \times 10^{2}$ c.f.u. of a mid-exponential-phase culture in a $25 \mu \mathrm{l}$ volume and incubated at $30{ }^{\circ} \mathrm{C}$ for $1 \mathrm{~h}$ on an orbital shaker (225 r.p.m.). Reactions were performed in quadruplicate for each strain tested. A $50 \mu \mathrm{l}$ volume from each reaction was spread on THA, and a control plate was prepared at the initiation of the assay as a reference for starting c.f.u. Each experiment was repeated three times.

Resistance to oxidative killing. The $\Delta p d i$ mutant and WT S. iniae were grown to mid-exponential phase and diluted in PBS such that adding $100 \mu \mathrm{l}$ of culture to a 96-well plate delivered $2 \times 10^{5}$ c.f.u. per well. A $100 \mu \mathrm{l}$ volume of dilute $\mathrm{H}_{2} \mathrm{O}_{2}$ was then added to each well, bringing the final concentration to $0.03,0.06$ or $0.09 \%$. After $1 \mathrm{~h}$ incubation at $30{ }^{\circ} \mathrm{C}$, the reaction was quenched by the addition of $10 \mu \mathrm{l}$ of a $1000 \mathrm{U}$ catalase $\mathrm{ml}^{-1}$ solution in PBS. c.f.u. data from a control plate prepared at the initiation of the assay served as a reference for the $60 \mathrm{~min}$ time point. Each experiment was repeated three times.

AMP susceptibility assays. Mid-exponential-phase cultures of $S$. iniae were diluted in fresh THB, and $1 \times 10^{5}$ c.f.u. in a total volume of $180 \mu \mathrm{l}$ was added to replicate wells of a 96-well plate. Dilutions of AMPs were prepared in deionized $\mathrm{H}_{2} \mathrm{O}\left(\mathrm{dH}_{2} \mathrm{O}\right)$, and $20 \mu \mathrm{l}$ was added to experimental wells for a final concentration of $1.5 \mu \mathrm{M}$ moronecidin (Lauth et al., 2002) or $60 \mu \mathrm{M}$ polymyxin B (Sigma). $\mathrm{dH}_{2} \mathrm{O}$ alone was used as a negative control. For the measurement of antimicrobial killing kinetics, $25 \mu \mathrm{l}$ aliquots were taken from each well at various time points, serially diluted in PBS and plated on THA for surviving c.f.u. determination. Each experiment was performed in triplicate.

Lysozyme sensitivity assays. For the determination of the sensitivity of $S$. iniae to lysozyme, cultures were washed once in $\mathrm{PBS}$, and resuspended in PBS to $\mathrm{OD}_{600}$ 0.4. Cultures were then diluted $1: 1000$ in DMEM (Gibco) plus 10\% FBS (Gibco) plus $20 \%$ $\mathrm{THB}$, and $180 \mu \mathrm{l}$ diluted bacteria $\left(3.6 \times 10^{4}\right.$ c.f.u. $)$ was added to a $96-$ well plate. A $20 \mu \mathrm{l}$ volume of chicken egg white lysozyme (Sigma) was added to a final concentration of $80 \mu \mathrm{g} \mathrm{ml}^{-1}$ to each well. Plates were incubated at $30{ }^{\circ} \mathrm{C}$ and serial dilutions were plated on THA after 30 min to enumerate surviving bacteria. To test sensitivity of growing cultures to lysozyme, the above procedure was followed, except that bacteria were allowed to grow for $1 \mathrm{~h}$ (sufficient time to reach exponential-phase growth) prior to adding lysozyme, incubating for $30 \mathrm{~min}$, and plating to enumerate surviving bacteria.

To test the sensitivity of a growing culture of $\Delta p d i S$. iniae to lysozyme, cultures were grown in DMEM/10\% FBS/20\% THB to mid-exponential phase $\left(\mathrm{OD}_{600} 0.4\right)$, and diluted $1: 20$ in the same medium in glass test tubes and incubated at $30{ }^{\circ} \mathrm{C}$. Growth was monitored by measuring the $\mathrm{OD}_{600}$ and lysozyme was added to a final concentration of $80 \mu \mathrm{g} \mathrm{ml} \mathrm{m}^{-1}$ during exponential-phase growth (around $\mathrm{OD}_{600}$ 0.1). All growth curves were conducted in triplicate.

Invasion and adherence assays. Invasion and adherence assays were carried out in 96-well collagenized plates (Nunc). One day prior to each assay, WBE27 cells were seeded in the wells at a density of $\sim 1 \times 10^{5}$ cells per well in $100 \mu$ DMEM $10 \%$ FBS medium and were allowed to grow to confluence overnight. Cell culture medium was removed, and $100 \mu \mathrm{lmid}$-exponential-phase bacterial cultures diluted 1:40 in DMEM 2\% FBS medium was added to confluent cells at an m.o.i. of 5 (bacteria to cells). The plate was then centrifuged at $350 \mathrm{~g}$ for $15 \mathrm{~min}$ to allow contact between bacteria and the cell monolayer. At 30 min post-centrifugation, the medium was removed, and the wells were washed four times with $200 \mu$ DMEM $2 \%$ FBS for the removal of any non-adherent bacteria. The cells were then lysed by trituration with $100 \mu \mathrm{l} 0.01 \%$ Triton X-100, and $25 \mu \mathrm{l}$ was removed, serially diluted and plated on THA for the enumeration of adherent c.f.u. Invasion assays were carried out in a similar manner, except that at $1 \mathrm{~h}$ post centrifugation, cell medium was removed, wells were washed once, and then were incubated with $150 \mu \mathrm{l}$ fresh DMEM containing $300 \mu \mathrm{g}$ gentamicin $\mathrm{ml}^{-1}$ (Invitrogen) and $30 \mu \mathrm{g}$ penicillin $\mathrm{ml}^{-1}$ (Invitrogen) for an additional hour. All strains of $S$. iniae were tested for susceptibility to these antibiotic concentrations at $1 \mathrm{~h}$. Cells were then washed three more times and lysed by trituration. Serial dilutions of lysed cell supernatant were plated on THA for the enumeration of surviving intracellular bacteria. Bacteria plated at the initiation of the assay and after $1 \mathrm{~h}$ in assay medium served as control references for adherence and invasion, respectively. 
Growth rate and autolysis analysis. $S$. iniae cultures were grown to mid-exponential phase and diluted 1:10 in THB in a 96-well plate. Eight replicates for each strain were monitored for growth over $12 \mathrm{~h}$ by the use of an EL808 plate reader and Gen5 software (BioTek Instruments). The incubation temperature was held constant at $30{ }^{\circ} \mathrm{C}$, and the plates were shaken before each reading (every $30 \mathrm{~min}$ ). Autolysis assays were performed as previously described (Kristian et al., 2005); an EL808 plate reader and Gen5 software were used to monitor decreases in OD.

Statistical analyses. Data resulting from in vitro assays were assessed by two-tailed unpaired $t$ tests. A $P$ value of $<0.05$ was considered to be statistically significant. In vitro assays were repeated at least three times, and the data presented (mean $\pm \mathrm{SE}$ ) are from single representative assays unless otherwise stated. Fish challenges were repeated with similar results. Statistical values obtained from mortality curves of in vivo challenges were generated from chisquared tests.

\section{RESULTS}

\section{Identification of S. iniae virulence gene pdi}

Using an HSB infection model, we identified the attenuated transposon mutant TnM7 from a Tn917 chromosomal insertion library of WT $S$. iniae strain K288, which had been created previously (Buchanan et al., 2005). TnM7 is one of approximately 40 attenuated $S$. iniae transposon mutants that were identified through initial virulence screening in HSB. To identify the gene or operon that had been disrupted by transposon insertion in the TnM7 chromosome, we used single-primer PCR (Karlyshev et al., 2000) to amplify genomic regions around the transposon insertion site and sequence the amplified DNA fragments.

The transposon insertion mapped immediately upstream of an ORF encoding a potential protein with high sequence similarity to bacterial polysaccharide deacetylases (by BLASTX analysis). We termed this putative gene and its predicted product $p d i$ and Pdi, respectively. The full sequence and chromosomal context of pdi were identified using BLASTN and a genomic library of contiguous sequence data generated from the pyrosequencing of the parent $S$. iniae strain K288 genome (Locke et al., 2008).

\section{Sequence analysis of Pdi}

Primary analysis of $S$. iniae Pdi showed that the protein possesses a probable signal peptide (SignalP probability = 0.875 ; cleavage probability $=0.716$ ), suggesting that it membrane-bound or, less likely, secreted. A motif search and protein family analysis using Interpro and Pfam revealed that Pdi, and its orthologues, share a deacetylase enzymic motif near the $\mathrm{C}$ terminus (Pfam Id Polysacc_deac_1 PF01522). This motif is also shared by the protein PgdA (Fig. 1b), which is highly conserved in many bacteria and has been well characterized for its role in virulence in S. pneumoniae (Vollmer \& Tomasz, 2000) and Listeria monocytogenes (Boneca et al., 2007). Using
PgdA sequence data from S. pneumoniae and Streptococcus pyogenes, a search of our S. iniae genomic library and the Baylor S. iniae genome project revealed a PgdA orthologue in S. iniae that is distinct from Pdi (Fig. 1), confirming that Pdi and PgdA are paralogous proteins. In fact, FigFam analysis revealed that Pdi and PgdA belong to two distinct protein families (FIG012537 and FIG015749, respectively), and that most streptococcal species whose genomes have been sequenced possess both proteins (Table 2).

Pdi homologues were found in other microbial genomes, and their sequences were aligned to the Pdi sequence (Fig. 1a) to infer a phylogenetic tree (Fig. 2). Pdi is conserved in the genomes of $S$. iniae phylogenetic neighbours (Table 2, Fig. 2). The conservation of its paralogue, PgdA, in all streptococci (Fig. 2) suggests that the proteins are not functionally redundant. Paralogues are often generated from gene duplication events that create variants of proteins with different substrate specificities or allosteric regulatory properties (Hooper \& Berg, 2003). Although there is extensive variability within and between the Pdi and PgdA family members (Figs 1 and 2), these homologues share highly conserved residues (Fig. 1).

\section{Targeted mutagenesis of the S. iniae pdi gene}

Upon successful generation of an isogenic $\Delta p d i$ mutant by precise in-frame allelic replacement of $p d i$ with cat in the WT S. iniae strain (Supplementary Fig. S1), we used the HSB i.p. challenge model to further evaluate the virulence of the newly created $\Delta p d i$ knockout. Significant attenuation was observed: no mortality occurred in fish challenged with the $\Delta p d i$ knockout at a dose that killed $70 \%$ of fish that received WT $(P=0.0011)$, and only $30 \%$ mortality occurred in fish challenged with the $\Delta p d i$ knockout at a dose that killed $90 \%$ of WT-injected fish $(P=0.0031)$ (Fig. 3).

\section{Effects of pdi deletion on S. iniae chain length and buoyancy}

WT S. iniae bacteria occur in short chains and are $\beta$ haemolytic on sheep blood agar (Fig. 4a and Supplementary Fig. S2b). The $\Delta p d i$ allelic exchange mutant lost its ability to maintain association in pairs or short chains, and cocci were seen predominantly as single cells (Fig. 4b). Expression of the pdi gene in the constitutive highexpression plasmid pDestErm was used to complement the $\Delta p d i$ knockout mutant and restored chain length (Fig. 4c).

Cultures of the $\Delta p d i$ mutant grown in liquid media exhibited greater buoyancy than the WT S. iniae parent strain (Supplementary Fig. S2a); the WT phenotype was partially restored in the plasmid-complemented strain. No differences in $\beta$-haemolytic activity of the strains were observed on sheep blood agar plates (Supplementary Fig. S2b). A similar growth profile was observed between the WT and the mutant through exponential phase $(P=0.6)$; 
(a) SEq_zOO_PODe $S P Y=M 1$ PODe SIn-UCSD PdI SAg_2603 $\bar{v}$ R POD LLa_la_Il $14403-P$ ECo_F11_PoDe BAn Ames PoDe SPn-R6_Pg dA SPY_M1-PgdA LLa_I1 1403 PgdA LMo_PgdA

consensus

SEq_zOO_PODe SPY_M1_PoDe SIn-UCSD PdI $S A g_{-} 2603 \overline{\mathrm{v}} \mathbf{R}_{-}$POD LLa_la_I $1 \overline{1} 4 \overline{0}_{3}$ P SAu_aureus_N315 ECo-F11_PoDe BAn_Ames_PoDe SPn_R6_P gdA SPY_M1-PgdA

LLa_I11403_PgdA LMo_PgdA consensus

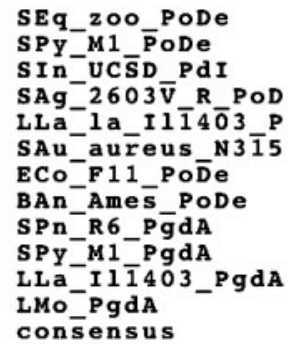

(b)
NLIVAPDIFEsqLKaMKEaGYYFLSpeEayrVLs an--------------ElPaeKV NLIVnPDIFDqqLqkMKDeGYYFLSPeEvYraLsnn------------ElPakKV NLIVAPDVFEShIKqLSEqGYYFLSPeEayrVLskn--------------EIPsdKV

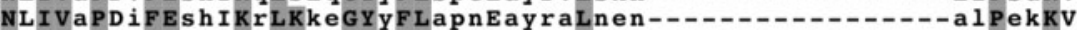
NnIn-ivtFEneLKaLKEkGYttVSgsDaekILttk------------EkPsdKM NysVsqsqFEsq I KWLKshdakFLTIkEf lyykkkg-stttsvraFnnqMawLRDrGYatLSmaqlegyVknk------------inlpaRa -LYVPPEqFEkhMqYLKDnGY tmLT ferwndInrvn--QIILyPspvvenLeeIalpvsaFfdviqssyLLekdaal-_-.-----yqsyfDkkhqKV snsLisDgFEvksgnLifdkklt Ipltt lfdvinpdflansdraaydnyrtykEqhpkKL kapyeksdkEa laKqVnadkqi I IerqkeaeLIanvqn-----------qYankKL eItnselilpvkIpgapEpnkvkVklaDiassvnkrylp-----ssvkvpevpkaktnkr nliv-pd-fe--1k-1ke-gy-fls--e---vl------------------e-p--kv

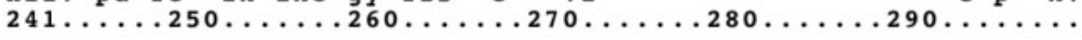

VWLTFDDs-mvdf YnvAFPILKKYdIKATNnVITGf teag----naanLT ldqMKEMKqa VWLTFDDs-midfYnvAYPILKKY aKATNnVITGltemg----saanLTlkqMKEMKqv VWLTFDDs-midfYrVAYPILKKY GaKATNnVITGftqnn----svanLTidqMKEMKkh IWITFDDG-nadfY tkAYPILKKYkVKATNnIITGfVqeg----resnLnvqqMIEMKqn VWLTFDDG-svtmY teiFPLLKKYnMhATNFIITGYvnkg----qggiLTweqIKEMKas VWInFDDm-detiYenAYPILKKYkIPATgFIITGhVgeenf-hnldmISkkeLKEMYkt VVITFDDG-1ksvsrYAYPVLKGYGMKATaFIVTsrIkrhpqkwnpksLqfmsVsELnei IF I TmDDG--rknnmnAlhILqKIkddt f QpaaTef L tan-eidkpnrLStdd I KqMds VaLTFDDGpnat tpqvietLaKYdIKATfFVLgknVsgn---.-----edlVKrIKse VaLTFDDGpdptt tpqv1dILaKYqaKGTfFMIgskVvnn---.------enltKrVsda IaLTFDDGpnpnttpqlikIf sdaqVpATfFaLgkqaqac-----------pqiIKEeadr I aLTFDDGpsasvtpgvidtLKRhnVKATfFVLgssViqn----------pglvKrelee vwltfDDg----y--afpilkky-ikatnfvitg-v---------1t--mkemk-$301 \ldots \ldots 310 \ldots \ldots 320 \ldots \ldots 330 \ldots \ldots 340 \ldots \ldots$.

-GMs -FQdHTvnHPdLSq--------aSa s st aEMkdSKeyLDReLhqdT I a IAYPaGr -GMs-FQdHTvnHPdLeq--------aSpdvQt tEMkdSKdyLDKqLnqnTIaIAYPs Gr -GMs-FOdHTvnHPdLSq-------qnevtonfEMqdSme fLDKeLsqkTI a IAcPaGr -GMs-FQgHTvtHPnLS1-------1TpelotqEMt 1 SKq fLDqkLs qdTLaIAYPsGr -GLvdFgSHTvSHPdLgk--------1TleaQrtELeqSKadLDKnLeqkTdi ICYPaGg -GLWeFeTHThd IhnLSknnkslmkaSeatiikDLnkSekyLtKnfkksktIAYPYG1 rdVfdFQSHThfihridgYrrpilisrsehnilfDfarSRraLaq-fnphVLYLSYPFg -GIf S iQSHTanHtmMah----------snnydeELrgSKekIEaltgkkvIaLAYPvGs --ghvvgnHSwsHPiLSq--------1S1deakkqI tdTedvLtKvLgssSk1MrpPYGa --gheianHTwdHPnLTn-------1SvseiqhqVnmTnqaIEKacgkkpryLrpPYGa --gnevasHTwdHkdLvt--------1 SpdqQkqEI esanqIInKitgknvt If rpPys

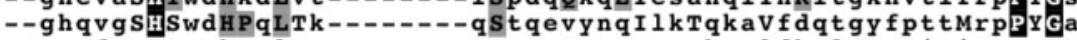
-gm--fqsHt--hp-ls----------s---q--em--sk--1dk-1---ti-iayPyG$361 \ldots \ldots 370 \ldots \ldots 380 \ldots \ldots 390 \ldots \ldots 400 \ldots \ldots 410 \ldots \ldots \ldots$

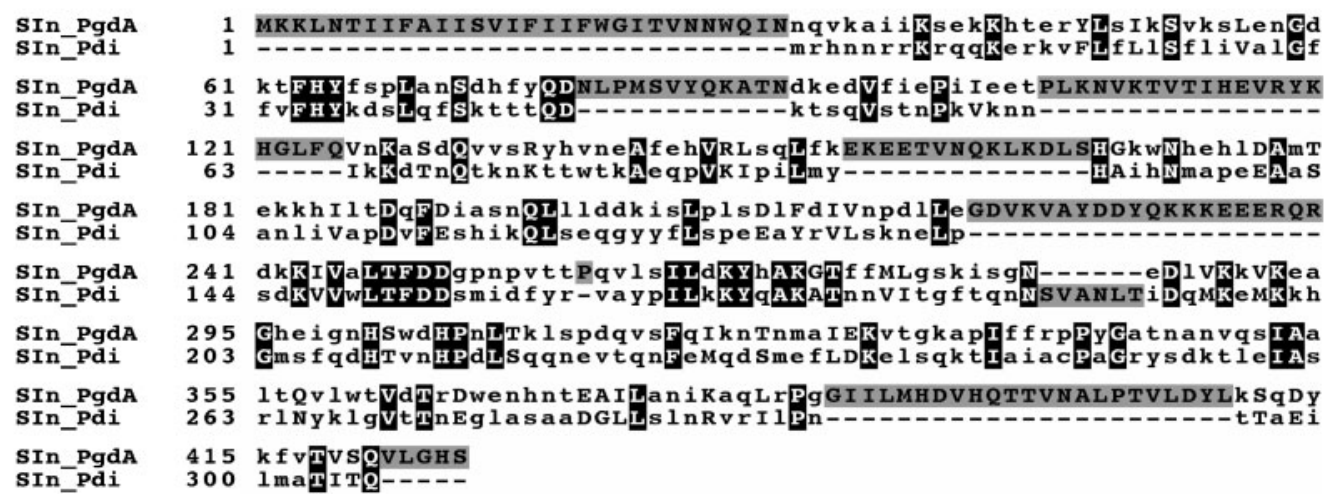

Fig. 1. Alignment of S. iniae Pdi and closely related proteins. These representations depict orthologues of Pdi found in other species as well as paralogous peptidoglycan deacetylase proteins (PgdA). (a) Amino acid alignment of conserved regions between polysaccharide deacetylases and peptidoglycan deacetylases that are found in multiple species. A full list of abbreviations is provided in Table 1. (b) Alignment of the predicted S. iniae Pdi and PgdA amino acid sequences shows low overall similarity but highly conserved patches, suggesting that the two proteins are paralogous and that Pdi has novel functional features in addition to the deacetylase motif shared with PgdA.

thus, no apparent gross defect in viability or fitness is conferred from loss of the pdi gene (Supplementary Fig. S2c). Phenotypes of impaired chain formation and differences in culture buoyancy of $\Delta p d i$ imply that the activity of Pdi involves alteration of molecules on the cell surface of S. iniae.

\section{Effect of pdi mutation on S. iniae susceptibility to lysozyme killing}

We found that the $S$. iniae $\Delta p d i$ mutant was significantly ( $P=0.0005)$ more sensitive to lysozyme than WT (Fig. 5a). In a manner similar to that reported for deacetylase 
Table 1. Abbreviations used in the alignments and phylogenetic analysis

\begin{tabular}{|ll|}
\hline Abbreviation & \multicolumn{1}{c|}{ Definition } \\
\hline ECo & Escherichia coli \\
SAu & Staphylococcus aureus \\
SEp & Staphylococcus epidermidis \\
SIn & Streptococcus iniae \\
SPy & Streptococcus pyogenes \\
SPn & Streptococcus pneumoniae \\
SAg & Streptococcus agalactiae \\
SEq & Streptococcus equi \\
STh & Streptococcus thermophilus \\
SMu & Streptococcus mutans \\
SSa & Streptococcus sanguinis \\
SSu & Streptococcus suis \\
SUb & Streptococcus uberis \\
SMi & Streptococcus mitis \\
SGo & Streptococcus gordonii \\
BAn & Bacillus anthracis \\
BCe & Bacillus cereus \\
LLa_la & Lactococcus lactis subsp. lactis \\
LMo & Listeria monocytogenes \\
PoDe & Polysaccharide deacetylase \\
PgdA & Peptidoglycan deacetylase \\
IcaB & PIA biosynthesis deacetylase \\
Pdi & Polysaccharide deacetylase of S. iniae \\
\hline
\end{tabular}

mutants in other bacterial species, we observed markedly reduced sensitivity in cells in exponential-phase growth (Boneca et al., 2007; Psylinakis et al., 2005; Vollmer \& Tomasz, 2000) (Fig. 5a, b).

Table 2. Distribution of $p g d A$ and pdi homologues in streptococcal genomes

The list was compiled through subsystem-based genome analysis (Overbeek et al., 2005) in the NMPDR database (McNeil et al., 2007). + , Present; -, absent.

\begin{tabular}{|lcc|}
\hline Genome & pod/pdi & pgdA \\
\hline S. pyogenes & + & + \\
S. equi & + & + \\
S. agalactiae & + & + \\
S. suis & + & + \\
S. thermophilus & + & + \\
S. uberis & + & + \\
S. iniae & + & + \\
S. gordonii & + & + \\
S. sanguinis & + & + \\
S. pneumoniae & - & + \\
S. mitis & - & + \\
\hline
\end{tabular}

\section{No difference in autolytic potential between S. iniae WT and the $\Delta$ pdi mutant}

We recognized the possibility that the virulence attenuation observed in HSB could be due to a greater propensity for autolysis in the $\Delta p d i$ mutant versus WT S. iniae; however, our experiments showed that the rates of autolysis for the two strains in a standardized assay did not differ significantly $(P=0.95)$ (Supplementary Fig. S3).

\section{S. iniae pdi promotes survival in blood}

To investigate additional possible mechanisms for the in vivo attenuation of the $\Delta p d i$ mutant, we assessed the ability of the $\Delta p d i$ mutant to survive in fresh fish blood. The mutant was significantly less able to survive in whole blood than WT $(P<0.002)$, while the complemented strain significantly restored whole blood survival to levels similar to those of the WT $(P<0.01)$ (Fig. 6).

\section{S. iniae pdi does not confer resistance to oxidative killing or cationic AMPs}

Reactive oxygen species and AMPs are mechanisms used by phagocytes and other immune cell types to mediate clearance of bacterial invaders in whole blood (Gallo \& Nizet, 2003; Locke et al., 2007a; Soehnlein et al., 2008). To further analyse why the $\Delta p d i$ mutant was less able to survive in whole fish blood, we compared the resistance of $\mathrm{WT}$ and the $\Delta p d i$ mutant to oxidative killing by hydrogen peroxide, as well as the sensitivities of both strains to the HSB-derived AMP moronecidin or the bacterially derived cationic AMP polymyxin B. We found no significant difference $(P=0.8)$ in the susceptibility to hydrogen peroxide or AMP killing between the two strains (Supplementary Fig. S4a-c). These studies imply that the increased susceptibility of the $\Delta p d i$ mutant to whole blood killing is not derived from enhanced sensitivity to the innate immune system oxidative killing mechanisms or AMPs.

\section{S. iniae $\Delta p d i$ mutant shows an impaired ability to adhere to and invade epithelial cells}

Compared with the WT parent strain, we found the $\Delta p d i$ mutant to be significantly reduced, by approximately twofold, in its ability to adhere to $(P<0.02)$ and invade $(P<0.007)$ fish epithelial cells (white bass epithelial cell line WBE27) (Fig. 7).

\section{DISCUSSION}

Enzymes that deacetylate polysaccharide molecules on the surface of bacterial cells have only recently been appreciated as virulence factors for various pathogenic species. Staphylococcus epidermidis uses the surface-attached polysaccharide deacetylase enzyme IcaB to deacetylate poly- $N$ acetylglucosamine (GlcNAc) residues of the polysaccharide 


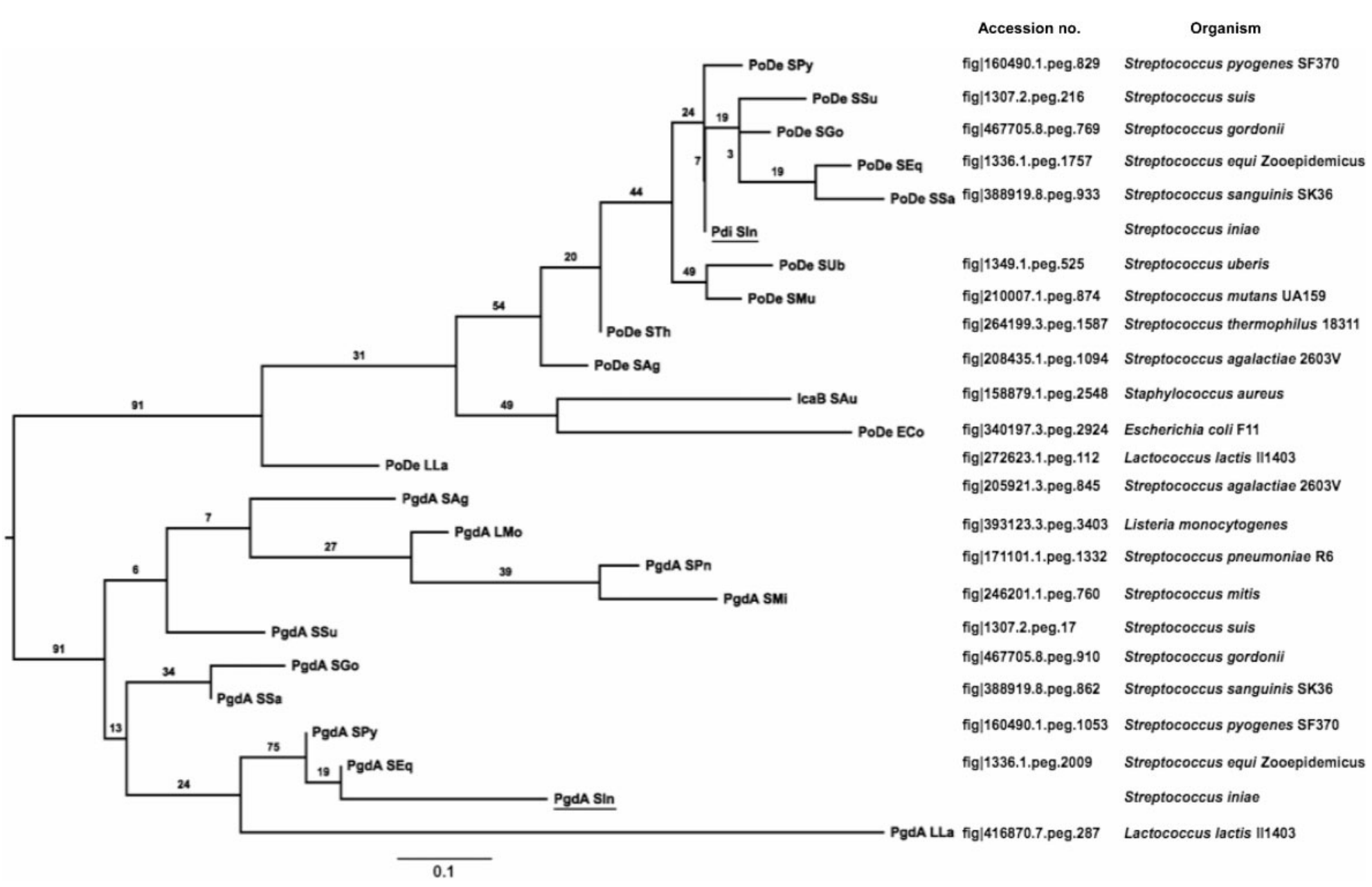

Fig. 2. Phylogenetic analysis of Pdi homologues. A maximum-likelihood tree produced by MUSCLE alignment followed by PhyML analysis (http://www.phylogeny.fr) with 100 bootstrap tests. These bootstrap values are shown as branch labels. The tree shows two major clusters, one of PgdA orthologues and another of Pdi orthologues. S. iniae Pdi and PgdA are underlined. 


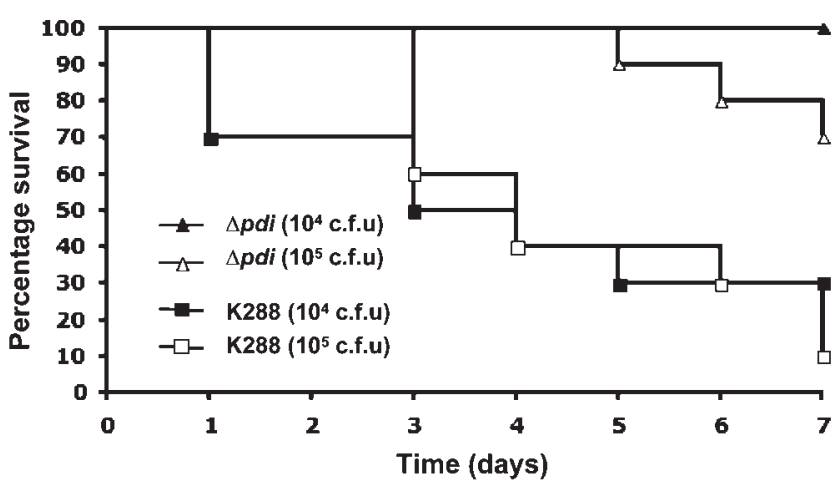

Fig. 3. Attenuated virulence of the $S$. iniae isogenic $\Delta p d i$ mutant in an HSB model. The $\Delta$ pdi mutant or WT bacteria were injected intraperitoneally at two doses ( $n=10$ per treatment). The mutant strain was significantly less virulent than WT at doses of both $1 \times 10^{4}(P=0.0011)$ and $1 \times 10^{5}(P=0.0031)$ c.f.u.

intercellular adhesin (PIA) molecule. The deacetylation of PIA promotes virulence mechanisms of Staphylococcus epidermidis such as biofilm formation, surface colonization and resistance to human AMPs (Vuong et al., 2004). In S. pneumoniae and Listeria monocytogenes, mutagenesis studies have revealed that cell surface peptidoglycan deacetylaces ( $p g d A s)$ play a role in virulence (Boneca et al., 2007; Vollmer \& Tomasz, 2002). The PgdA enzymes are metal-dependent deacetylases and members of the family 4 carbohydrate esterases (Blair et al., 2005). In S. pneumoniae, PgdA has been shown to de- $N$-acetylate GlcNAc residues of the peptidoglycan cell wall and provide resistance to the activity of lysozyme (Vollmer \& Tomasz, 2000, 2002). Additionally, PgdA has been reported to promote survival of $L$. monocytogenes in macrophages and also to confer resistance to host lysozyme (Boneca et al., 2007). These results indicate a possible role for deacetylation as a general mechanism for evasion of host innate immune responses, primarily through modification of GlcNAc residues.

Through our studies of our S. iniae Tn917 insertion mutant library (Buchanan et al., 2005), we discovered mutant TnM7 with attenuated virulence in an HSB model of fish meningoencephalitis. Due to the unavailability of a fully assembled $S$. iniae genome sequence, we used a genomic library that we created by pyrosequencing $S$. iniae strain K288, and were able to map the transposon insertion of TnM7 to a location upstream of a putative polysaccharide deacetylase gene ( $p d i)$ in the $S$. iniae chromosome. Through phylogenetic and protein family analyses, we have concluded that $S$. iniae $p d i$ is a paralogue of the highly conserved Grampositive $p g d A$ gene, but that Pdi and PgdA are likely not redundant. Although the two predicted proteins contain the same motif (Pfam PF01522) and share conserved amino acid residues, they belong to different protein families (FIG012537 and FIG015749, respectively). Moreover, while $S$. pneumoniae genomes only have PgdA orthologues, the $S$. iniae genome contains the two paralogues, $p d i$ and $p g d A$. The conservation of Pdi in the genomes of bacteria closely related to $S$. iniae suggests that this protein is ancestral in streptococci rather than horizontally acquired, and the conservation of its paralogue, PgdA, in all streptococci suggests that the proteins are not functionally redundant. Paralogues are often generated from gene duplication events that create variants of proteins with different substrate specificities or allosteric regulatory properties (Hooper \& Berg, 2003). Taken together, our in silico analyses of the similarity and variability among Pdi and PgdA homologues imply that: (1) while Pdi and PgdA are two distinct paralogues, they share a molecular motif that is most likely responsible for their deacetylase enzymic activity; (2) while $p d i$ is a gene likely to be responsible for the production of a deacetylase with some sequence similarity to PgdA, it is in fact a novel gene that has yet to be characterized. Thus, any identified roles of the $S$. iniae pdi gene in virulence could be divergent and distinct from those attributed to $p g d A$. Based on these results, $p d i$ and $p g d A$ may both have the capacity to play unique roles in the virulence of other streptococcal pathogens that contain both genes, such as S. pyogenes and Streptococcus agalactiae.

Observations of morphological differences between the isogenic $\Delta p d i$ mutant and WT strain provide strong circumstantial evidence that pdi works on the cell surface
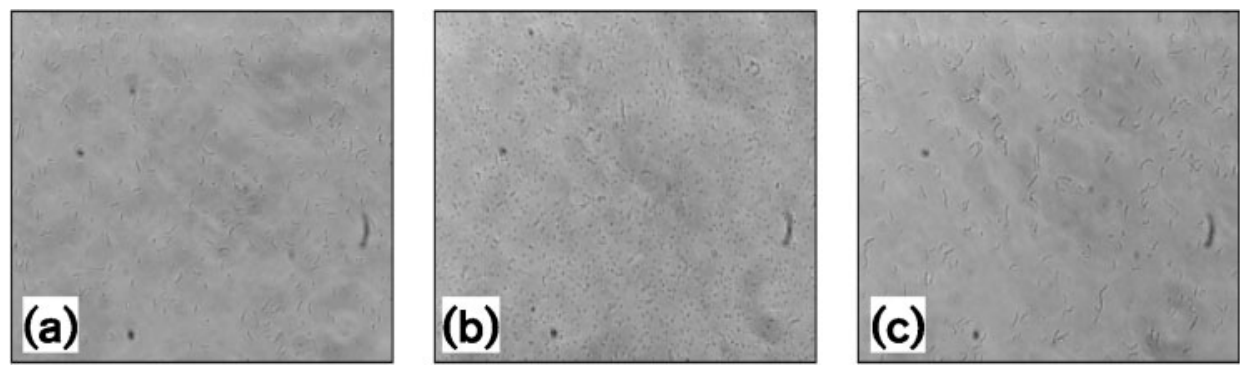

Fig. 4. The $\Delta p d i$ mutant lacks the ability to remain in chains. Photomicrographs taken of early stationary phase cultures $\left(O D_{600}\right.$ 0.8 ) indicate that the $S$. iniae $\Delta$ pdi mutant is impaired in its ability to remain in chains. In (a), WT, most bacteria appear in short chains, while in (b), mutant, most bacteria exist as single cocci. In (c), the mutant is complemented to restore the WT phenotype. 

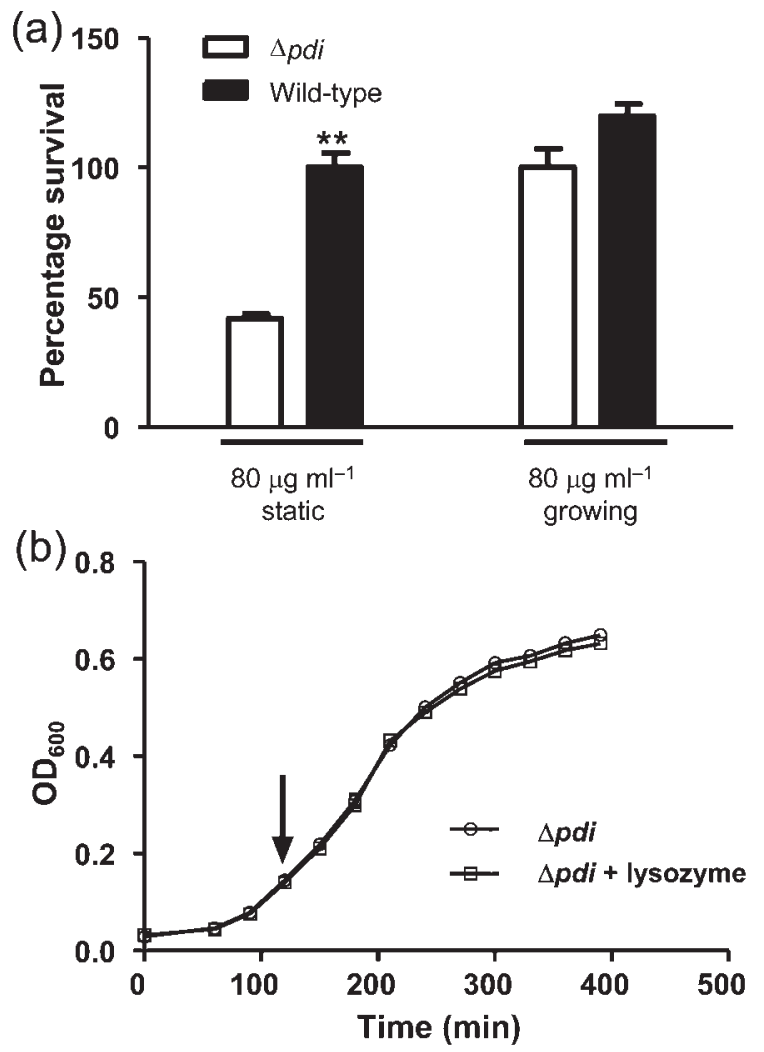

Fig. 5. The $S$. iniae $\Delta p d i$ mutant is more susceptible to lysozyme than WT. (a) Observed differences in the kinetic killing of WT (black bars) and the $\Delta$ pdi mutant (white bars) by lysozyme show that the mutant is significantly more susceptible to lysozyme killing when static cells are exposed $\left({ }^{* *} P=0.0005\right)$, but significant differences were not detected when cells were dividing $(P=0.0803)$. Each experiment was performed with exposure to $80 \mu \mathrm{g}$ lysozyme $\mathrm{ml}^{-1}$ for $30 \mathrm{~min}$. Values plotted are mean $\pm \mathrm{SEM}$. (b) Addition of $80 \mu \mathrm{g}$ lysozyme $\mathrm{ml}^{-1}$ to an exponential-phase culture of the $\Delta p d i$ mutant does not result in killing. The arrow indicates the time point when lysozyme was added to the culture. Values plotted are mean \pm SEM.

of S. iniae. The $\Delta p d i$ mutant has an impaired ability to form chains characteristic of the WT $S$. iniae strain, and its cells are predominantly found as single cocci. We believe that this phenotype contributes to the observed increased buoyancy in liquid media, since longer chains are more likely to settle out of solution. Because complementation of the mutant restores both of these phenotypes, we hypothesize that the increased buoyancy of $\Delta p d i$ liquid cultures and the inability to form chains are due to the loss of Pdi function at the $S$. iniae cell surface. A peptidoglycan deacetylase in Lactococcus lactis that affects chain length morphology and resistance to lysozyme killing also protects against cell autolysis (Meyrand et al., 2007). However, we were unable to detect any differences in autolysis.

The attenuated virulence of the $\Delta p d i$ mutant observed in the HSB infection model is reflected in the decreased ability

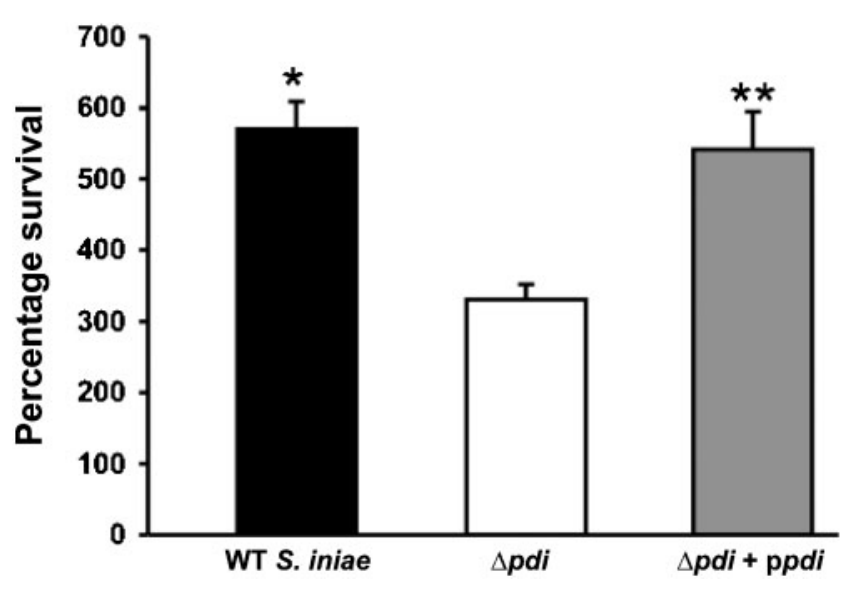

Fig. 6. The $S$. iniae $\Delta p d i$ mutant survives significantly less well than WT in whole fish blood. The WT and $\Delta$ pdi mutant were incubated with pooled blood from HSB fingerlings (average $n=5$ ). The $\Delta$ pdi mutant showed a significantly reduced ability to survive in whole fish blood compared with WT ${ }^{*}(P<0.002)$, with survival being restored to levels similar to those of the WT in the complemented strain, which were significantly greater than those in the $\Delta$ pdi mutant ${ }^{* *}(P<0.01)$. Values are mean \pm SEM.

of the mutant to survive in whole blood. Gene deletion strains of $S$. iniae, $S$. pyogenes or $S$. agalactiae with diminished ability to survive in fresh blood show reduced animal virulence (Buchanan et al., 2005; Datta et al., 2005; Liu et al., 2004; Locke et al., 2007a). These results suggest a fitness advantage conferred by the $S$. iniae pdi gene against immune clearance by cells or soluble components present in the fish blood circulation. One possible explanation is that the Pdi-dependent ability of $S$. iniae to form longer chains reduces the efficiency of phagocytic uptake and clearance. Another possible explanation for this decreased survival is that deacetylation of $S$. iniae cell surface molecules introduces significant changes in molecular patterns that are otherwise easily recognized by the host immune system, thus allowing WT S. iniae to survive and persist in vivo (Arancibia et al., 2007; Nigro et al., 2008). In examining specific potential effectors of phagocytic killing, we have found no difference in the kinetics of the killing of WT and $\Delta p d i$ by hydrogen peroxide (a component of the oxidative burst) and the cationic HSB AMP moronecidin.

Resistance to cell wall hydrolysis by lysozyme has been reported as a key protective role of the peptidoglycan deacetylases $p g d A$ in bacterial species such as Bacillus cereus (Psylinakis et al., 2005), S. pneumoniae (Vollmer \& Tomasz, 2000) and L. monocytogenes (Boneca et al., 2007). Notably, pgdA mutants are sensitive to lysozyme killing primarily in stationary phase and are resistant when cells are actively growing. Similarly, $S$. iniae $\Delta p d i$ mutants express an increased sensitivity to the enzymic activity of lysozyme when not growing, but $\Delta p d i$ mutant cells in growth phase are apparently no more resistant to lysozyme killing. Yet, while the $S$. iniae $\Delta p d i$ mutant is significantly 

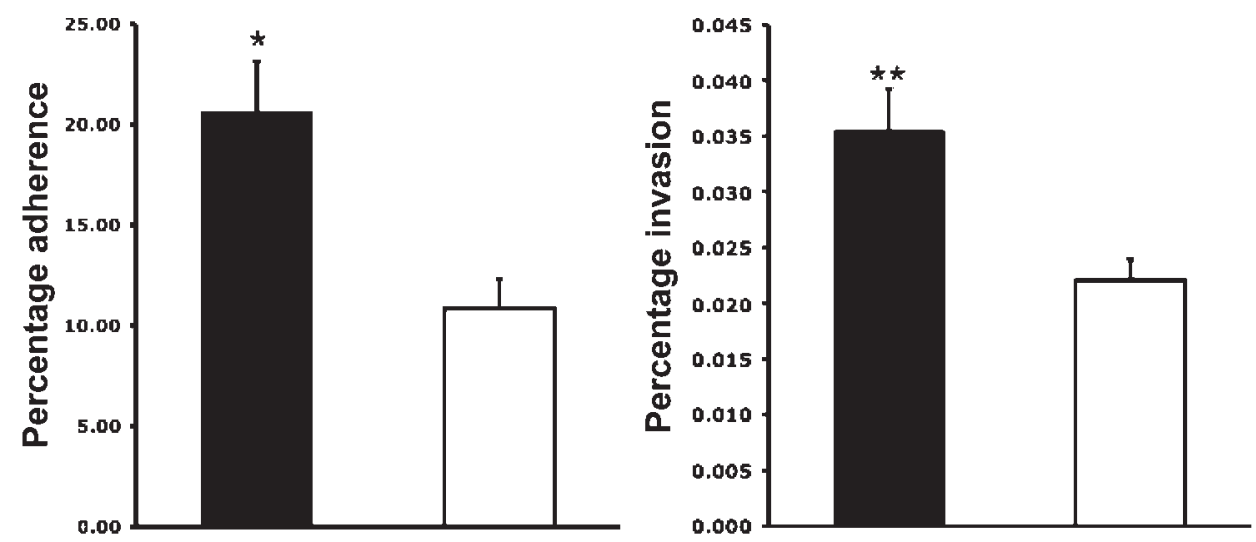

Fig. 7. Adherence to and invasion of white bass epithelial cell line WBE27 by S. iniae WT and $\Delta$ pdi mutant. Adherence and invasion of innate immunity barriers, such as epithelial cells and the blood-brain barrier are thought to be keys for virulence in many streptococcal species. The $S$. iniae $\Delta$ pdi mutant (white bars) was observed to have a significantly reduced ability to adhere to ${ }^{*}(P<0.02)$ and invade ${ }^{* *}(P<0.007)$ WBE27 cells compared with WT (black bars). Values are mean \pm SEM.

(threefold) more susceptible to lysozyme than WT, the differences are not nearly as great as the three-log difference observed between WT and deacetylase mutants of other pathogenic species (Boneca et al., 2007). This is likely the result of $S$. iniae possessing a functional PgdA; therefore, deacetylase activity in the $\Delta p d i$ mutant is only reduced, not abolished. We hypothesize that while the decreased resistance to lysozyme of the isogenic $\Delta p d i$ mutant is important, this does not fully account for the attenuated virulence. It is important to note that we used lysozyme from a non-host species (chicken) in these assays, and it is possible that different results would be observed in similar experiments performed using fish lysozyme.

The impaired ability of the $\Delta p d i$ mutant to adhere to and invade host cells may indicate a novel role for $p d i$ in $S$. iniae virulence. To date, no association between a polysaccharide deacetylase of a pathogenic bacterial species and the ability of an organism to bind to or enter host cells has been described. Adherence to and invasion of innate immunity barriers, such as epithelial cells and the blood-brain barrier, are thought to be keys to virulence for many streptococcal species, including S. iniae (Eyngor et al., 2007; Locke et al., 2007b; Rajam et al., 2008; Williamson et al., 2008). It has recently been shown that transcytosis across host cell barriers may be a key virulence mechanism of $S$. iniae, for which adherence to and invasion of host cells would play an integral role (Eyngor et al., 2007). Fish mortality associated with $S$. iniae infection is most often attributed to fatal menigoencephalitis (Agnew \& Barnes, 2007; Locke et al., 2007b), in which the bacterial agent must cross the blood-brain barrier to cause the fatal pathology of this disease. Although our HSB model of virulence in fish was conducted by i.p. injection, the impaired ability of the $\Delta p d i$ mutant to adhere to and invade host cells may render the bacteria less able to initiate the proper contact needed for dissemination and entry into a variety of tissue microenvironments, including the central nervous system (Zlotkin et al., 2003). In addition, the reduction of this virulence mechanism may leave the bacteria more susceptible to unfavourable host conditions and cause the bacteria to forfeit the ability to mechanically hide from host defences (Eyngor et al., 2007), thus contributing largely to the attenuation witnessed in ex vivo and in vivo experiments. Apparently, removal of deacetylation activity in the $\Delta p d i$ mutant interferes with certain steps in the pathogenic process by decreasing attachment to and invasion of epithelial cells, while simultaneously increasing sensitivity to host lysozyme and clearance by host phagocytes. In our fish infection challenge, these phenomena may play a critical role in determining the outcome of infection.

In summary, as a product of high-throughput genomewide approaches, we discovered a putative cell surface polysaccharide deacetylase gene ( $p d i)$ that we suggest plays a novel role in $S$. iniae fish virulence. The predicted amino acid sequence of the pdi gene product contains active residues similar to other well-studied cell surface deacetylases, and through allelic-replacement mutagenesis we have shown that $p d i$ affects morphological phenotypes of $S$. iniae and plays a role in resistance to lysozyme killing. We propose that the sensitivity of the $\Delta p d i$ mutant to lysozyme, and its shortened chain length, impaired ability to survive in whole blood and impaired ability to adhere to and invade host cells, indicate mechanisms of pathogenesis associated with this gene and define Pdi as a novel and significant virulence factor for $S$. iniae infection. Because of its predicted membrane-localization signal peptide, Pdi itself may function as an adhesin that directly interacts with host cell receptors. As the true polysaccharide substrate of $p d i$ is not known, we propose that in addition to its own role, it could be involved in the post-transcriptional regulation of other virulence molecules, which may 
themselves confer an ability to adhere to and invade host cells. This virulence factor is of interest as a recombinant vaccine target or for the development of attenuated $\Delta p d i$ strains for vaccination programmes in the aquaculture industry.

\section{ACKNOWLEDGEMENTS}

We thank Kent SeaTech Corporation for the use of their fish and challenge facilities. We thank Dr Robert A. Edwards for his assistance with processing the 454 sequence data. This publication was supported in part by the National Sea Grant College Program of the US Department of Commerce's National Oceanic and Atmospheric Administration under NOAA Grant \#NA04OAR4170038, project \#59-A-N, through the California Sea Grant College Program; and in part by the California State Resources Agency. The views expressed herein do not necessarily reflect the views of any of those organizations. Additional funding was provided by the NIH Training Program in Marine Biotechnology grant \#5 T32 GM067550. The DNA sequence of $S$. iniae strain 9117 was supported by USDA grant \#2006-35600-16569 at the Baylor College of Medicine Human Genome Sequencing Center (BCM-HGSC).

\section{REFERENCES}

Agnew, W. \& Barnes, A. C. (2007). Streptococcus iniae: an aquatic pathogen of global veterinary significance and a challenging candidate for reliable vaccination. Vet Microbiol 122, 1-15.

Altschul, S. F., Madden, T. L., Schaffer, A. A., Zhang, J., Zhang, Z., Miller, W. \& Lipman, D. J. (1997). Gapped BLAST and PSI-BLAST: a new generation of protein database search programs. Nucleic Acids Res 25, 3389-3402.

Apweiler, R., Attwood, T. K., Bairoch, A., Bateman, A., Birney, E., Biswas, M., Bucher, P., Cerutti, L., Corpet, F. \& other authors (2001). The InterPro database, an integrated documentation resource for protein families, domains and functional sites. Nucleic Acids Res 29, $37-40$.

Arancibia, S. A., Beltran, C. J., Aguirre, I. M., Silva, P., Peralta, A. L., Malinarich, F. \& Hermoso, M. A. (2007). Toll-like receptors are key participants in innate immune responses. Biol Res 40, 97-112.

Aziz, R. K., Bartels, D., Best, A. A., DeJongh, M., Disz, T., Edwards, R. A., Formsma, K., Gerdes, S., Glass, E. M. \& other authors (2008). The RAST server: rapid annotations using subsystems technology. BMC Genomics 9, 75.

Baiano, J. C., Tumbol, R. A., Umapathy, A. \& Barnes, A. C. (2008). Identification and molecular characterisation of a fibrinogen binding protein from Streptococcus iniae. BMC Microbiol 8, 67.

Barnes, A. C., Young, F. M., Horne, M. T. \& Ellis, A. E. (2003). Streptococcus iniae: serological differences, presence of capsule and resistance to immune serum killing. Dis Aquat Organ 53, 241-247.

Bendtsen, J. D., Nielsen, H., von Heijne, G. \& Brunak, S. (2004). Improved prediction of signal peptides: SignalP 3.0. J Mol Biol 340, 783-795.

Blair, D. E., Schuttelkopf, A. W., MacRae, J. I. \& van Aalten, D. M. (2005). Structure and metal-dependent mechanism of peptidoglycan deacetylase, a streptococcal virulence factor. Proc Natl Acad Sci U S A 102, 15429-15434.

Boneca, I. G., Dussurget, O., Cabanes, D., Nahori, M. A., Sousa, S., Lecuit, M., Psylinakis, E., Bouriotis, V., Hugot, J. P. \& other authors (2007). A critical role for peptidoglycan $N$-deacetylation in Listeria evasion from the host innate immune system. Proc Natl Acad Sci U S A 104, 997-1002.

Buchanan, J. T., Stannard, J. A., Lauth, X., Ostland, V. E., Powell, H. C., Westerman, M. E. \& Nizet, V. (2005). Streptococcus iniae phosphoglucomutase is a virulence factor and a target for vaccine development. Infect Immun 73, 6935-6944.

Buchanan, J. T., Simpson, A. J., Aziz, R. K., Liu, G. Y., Kristian, S. A., Kotb, M., Feramisco, J. \& Nizet, V. (2006). DNase expression allows the pathogen group A Streptococcus to escape killing in neutrophil extracellular traps. Curr Biol 16, 396-400.

Datta, V., Myskowski, S. M., Kwinn, L. A., Chiem, D. N., Varki, N., Kansal, R. G., Kotb, M. \& Nizet, V. (2005). Mutational analysis of the group A streptococcal operon encoding streptolysin $\mathrm{S}$ and its virulence role in invasive infection. Mol Microbiol 56, 681-695.

Dereeper, A., Guignon, V., Blanc, G., Audic, S., Buffet, S., Chevenet, F., Dufayard, J. F., Guindon, S., Lefort, V. \& other authors (2008). Phylogeny.fr: robust phylogenetic analysis for the non-specialist. Nucleic Acids Res 36, W465-W469.

Eyngor, M., Chilmonczyk, S., Zlotkin, A., Manuali, E., Lahav, D., Ghittino, C., Shapira, R., Hurvitz, A. \& Eldar, A. (2007). Transcytosis of Streptococcus iniae through skin epithelial barriers: an in vitro study. FEMS Microbiol Lett 277, 238-248.

Facklam, R., Elliott, J., Shewmaker, L. \& Reingold, A. (2005). Identification and characterization of sporadic isolates of Streptococcus iniae isolated from humans. J Clin Microbiol 43, 933-937.

Felsenstein, J. (1997). An alternating least squares approach to inferring phylogenies from pairwise distances. Syst Biol 46, 101-111.

Finn, R. D., Tate, J., Mistry, J., Coggill, P. C., Sammut, S. J., Hotz, H. R., Ceric, G., Forslund, K., Eddy, S. R. \& other authors (2008). The Pfam protein families database. Nucleic Acids Res 36, D281-D288.

Fuller, J. D., Camus, A. C., Duncan, C. L., Nizet, V., Bast, D. J., Thune, R. L., Low, D. E. \& De Azavedo, J. C. (2002). Identification of a streptolysin S-associated gene cluster and its role in the pathogenesis of Streptococcus iniae disease. Infect Immun 70, 5730-5739.

Gallo, R. L. \& Nizet, V. (2003). Endogenous production of antimicrobial peptides in innate immunity and human disease. Curr Allergy Asthma Rep 3, 402-409.

Gordon, D., Abajian, C. \& Green, P. (1998). Consed: a graphical tool for sequence finishing. Genome Res 8, 195-202.

Higgins, D. G., Thompson, J. D. \& Gibson, T. J. (1996). Using CLUSTAL for multiple sequence alignments. Methods Enzymol 266, 383-402.

Hooper, S. D. \& Berg, O. G. (2003). On the nature of gene innovation: duplication patterns in microbial genomes. Mol Biol Evol 20, 945-954.

Karlyshev, A. V., Pallen, M. J. \& Wren, B. W. (2000). Single-primer PCR procedure for rapid identification of transposon insertion sites. Biotechniques 28, 1078, 1080, 1082.

Kristian, S. A., Datta, V., Weidenmaier, C., Kansal, R., Fedtke, I., Peschel, A., Gallo, R. L. \& Nizet, V. (2005). D-Alanylation of teichoic acids promotes group A Streptococcus antimicrobial peptide resistance, neutrophil survival, and epithelial cell invasion. J Bacteriol 187, 6719-6725.

Lau, S. K., Woo, P. C., Tse, H., Leung, K. W., Wong, S. S. \& Yuen, K. Y. (2003). Invasive Streptococcus iniae infections outside North America. J Clin Microbiol 41, 1004-1009.

Lau, S. K., Woo, P. C., Luk, W. K., Fung, A. M., Hui, W. T., Fong, A. H., Chow, C. W., Wong, S. S. \& Yuen, K. Y. (2006). Clinical isolates of Streptococcus iniae from Asia are more mucoid and $\beta$-hemolytic than those from North America. Diagn Microbiol Infect Dis 54, 177-181.

Lauth, X., Shike, H., Burns, J. C., Westerman, M. E., Ostland, V. E., Carlberg, J. M., Van Olst, J. C., Nizet, V., Taylor, S. W. \& other authors (2002). Discovery and characterization of two isoforms of 
moronecidin, a novel antimicrobial peptide from hybrid striped bass. J Biol Chem 277, 5030-5039.

Liu, G. Y., Doran, K. S., Lawrence, T., Turkson, N., Puliti, M., Tissi, L. \& Nizet, V. (2004). Sword and shield: linked group B streptococcal $\beta$ hemolysin/cytolysin and carotenoid pigment function to subvert host phagocyte defense. Proc Natl Acad Sci U S A 101, 14491-14496.

Locke, J. B., Colvin, K. M., Datta, A. K., Patel, S. K., Naidu, N. N., Neely, M. N., Nizet, V. \& Buchanan, J. T. (2007a). Streptococcus iniae capsule impairs phagocytic clearance and contributes to virulence in fish. J Bacteriol 189, 1279-1287.

Locke, J. B., Colvin, K. M., Varki, N., Vicknair, M. R., Nizet, V. \& Buchanan, J. T. (2007b). Streptococcus iniae $\beta$-hemolysin streptolysin $S$ is a virulence factor in fish infection. Dis Aquat Organ 76, 17-26.

Locke, J. B., Aziz, R. K., Vicknair, M. R., Nizet, V. \& Buchanan, J. T. (2008). Streptococcus iniae M-like protein contributes to virulence in fish and is a target for live attenuated vaccine development. PLoS One 3, e2824.

Lowe, B. A., Miller, J. D. \& Neely, M. N. (2007). Analysis of the polysaccharide capsule of the systemic pathogen Streptococcus iniae and its implications in virulence. Infect Immun 75, 1255-1264.

McNeil, L. K., Reich, C., Aziz, R. K., Bartels, D., Cohoon, M., Disz, T., Edwards, R. A., Gerdes, S., Hwang, K. \& other authors (2007). The National Microbial Pathogen Database Resource (NMPDR): a genomics platform based on subsystem annotation. Nucleic Acids Res 35, D347-D353.

Meyrand, M., Boughammoura, A., Courtin, P., Mezange, C., Guillot, A. \& Chapot-Chartier, M. P. (2007). Peptidoglycan $N$-acetylglucosamine deacetylation decreases autolysis in Lactococcus lactis. Microbiology 153, 3275-3285.

Miller, J. D. \& Neely, M. N. (2005). Large-scale screen highlights the importance of capsule for virulence in the zoonotic pathogen Streptococcus iniae. Infect Immun 73, 921-934.

Nakano, Y., Yoshida, Y., Yamashita, Y. \& Koga, T. (1995). Construction of a series of pACYC-derived plasmid vectors. Gene 162, 157-158.

Nigro, G., Fazio, L. L., Martino, M. C., Rossi, G., Tattoli, I., Liparoti, V., De Castro, C., Molinaro, A., Philpott, D. J. \& Bernardini, M. L. (2008). Muramylpeptide shedding modulates cell sensing of Shigella flexneri. Cell Microbiol 10, 682-695.

Overbeek, R., Begley, T., Butler, R. M., Choudhuri, J. V., Chuang, H. Y., Cohoon, M., de Crécy-Lagard, V., Diaz, N., Disz, T. \& other authors (2005). The subsystems approach to genome annotation and its use in the project to annotate 1000 genomes. Nucleic Acids Res 33, 5691-5702.

Psylinakis, E., Boneca, I. G., Mavromatis, K., Deli, A., Hayhurst, E., Foster, S. J., Varum, K. M. \& Bouriotis, V. (2005). Peptidoglycan
$\mathrm{N}$-acetylglucosamine deacetylases from Bacillus cereus, highly conserved proteins in Bacillus anthracis. J Biol Chem 280, 30856-30863.

Rajam, G., Phillips, D. J., White, E., Anderton, J., Hooper, C. W., Sampson, J. S., Carlone, G. M., Ades, E. W. \& Romero-Steiner, S. (2008). A functional epitope of the pneumococcal surface adhesin A activates nasopharyngeal cells and increases bacterial internalization. Microb Pathog 44, 186-196.

Shimizu, C., Shike, H., Malicki, D. M., Breisch, E., Westerman, M., Buchanan, J., Ligman, H. R., Phillips, R. B., Carlberg, J. M. \& other authors (2003). Characterization of a white bass (Morone chrysops) embryonic cell line with epithelial features. In Vitro Cell Dev Biol Anim 39, 29-35.

Soehnlein, O., Kenne, E., Rotzius, P., Eriksson, E. E. \& Lindbom, L. (2008). Neutrophil secretion products regulate anti-bacterial activity in monocytes and macrophages. Clin Exp Immunol 151, 139-145.

Subramaniam, S. (1998). The Biology Workbench - a seamless database and analysis environment for the biologist. Proteins 32, 1-2.

Sun, J. R., Yan, J. C., Yeh, C. Y., Lee, S. Y. \& Lu, J. J. (2007). Invasive infection with Streptococcus iniae in Taiwan. J Med Microbiol 56, 1246-1249.

Thompson, J. D., Higgins, D. G. \& Gibson, T. J. (1994). CLUSTAL W: improving the sensitivity of progressive multiple sequence alignment through sequence weighting, position-specific gap penalties and weight matrix choice. Nucleic Acids Res 22, 4673-4680.

Vollmer, W. \& Tomasz, A. (2000). The pgdA gene encodes for a peptidoglycan $\mathrm{N}$-acetylglucosamine deacetylase in Streptococcus pneumoniae. J Biol Chem 275, 20496-20501.

Vollmer, W. \& Tomasz, A. (2002). Peptidoglycan $N$-acetylglucosamine deacetylase, a putative virulence factor in Streptococcus pneumoniae. Infect Immun 70, 7176-7178.

Vuong, C., Kocianova, S., Voyich, J. M., Yao, Y., Fischer, E. R., DeLeo, F. R. \& Otto, M. (2004). A crucial role for exopolysaccharide modification in bacterial biofilm formation, immune evasion, and virulence. J Biol Chem 279, 54881-54886.

Weinstein, M. R., Litt, M., Kertesz, D. A., Wyper, P., Rose, D., Coulter, M., McGeer, A., Facklam, R., Ostach, C. \& other authors (1997). Invasive infections due to a fish pathogen, Streptococcus iniae. N Engl J Med 337, 589-594.

Williamson, Y. M., Gowrisankar, R., Longo, D. L., Facklam, R., Gipson, I. K., Ades, E. P., Carlone, G. M. \& Sampson, J. S. (2008). Adherence of nontypeable Streptococcus pneumoniae to human conjunctival epithelial cells. Microb Pathog 44, 175-185.

Zlotkin, A., Chilmonczyk, S., Eyngor, M., Hurvitz, A., Ghittino, C. \& Eldar, A. (2003). Trojan horse effect: phagocyte-mediated Streptococcus iniae infection of fish. Infect Immun 71, 2318-2325.

Edited by: S. D. Bentley 